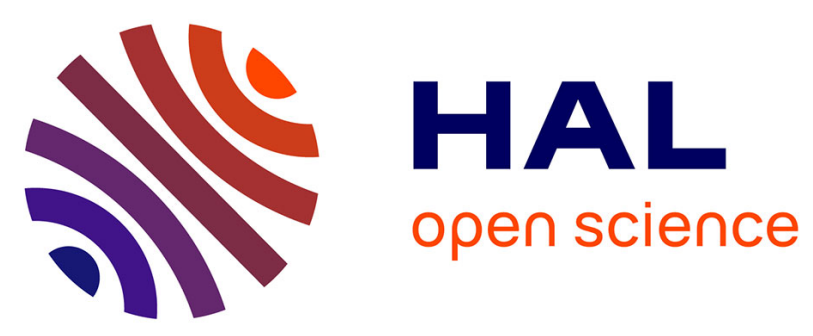

\title{
The early stage of formation of self-organized nanocolumns in thin films: Monte Carlo simulations versus atomic-scale observations in Ge-Mn
}

I. Mouton, Etienne Talbot, Cristelle Pareige, R. Larde, D. Blavette

\section{- To cite this version:}

I. Mouton, Etienne Talbot, Cristelle Pareige, R. Larde, D. Blavette. The early stage of formation of self-organized nanocolumns in thin films: Monte Carlo simulations versus atomic-scale observations in Ge-Mn. Journal of Applied Physics, 2014, 115 (5), 10.1063/1.4864271 . hal-01633479

\section{HAL Id: hal-01633479 \\ https://hal.science/hal-01633479}

Submitted on 28 Feb 2019

HAL is a multi-disciplinary open access archive for the deposit and dissemination of scientific research documents, whether they are published or not. The documents may come from teaching and research institutions in France or abroad, or from public or private research centers.
L'archive ouverte pluridisciplinaire HAL, est destinée au dépôt et à la diffusion de documents scientifiques de niveau recherche, publiés ou non, émanant des établissements d'enseignement et de recherche français ou étrangers, des laboratoires publics ou privés. 


\title{
The early stage of formation of self-organized nanocolumns in thin films: Monte Carlo simulations versus atomic-scale observations in Ge-Mn
}

\author{
I. Mouton, E. Talbot, ${ }^{a}{ }^{2}$ C. Pareige, R. Lardé, and D. Blavette \\ Groupe de Physique des Matériaux (GPM) UMR 6634, Normandie Université, Université et INSA \\ de Rouen-CNRS, Av. de l'Université, BP 12, 76801 Saint Etienne du Rouvray, France
}

(Received 12 November 2013; accepted 24 January 2014; published online 6 February 2014)

\begin{abstract}
Formation kinetics of self-organized nanocolumns during epitaxial growth of a thin film composed of immiscible elements (A,B) has been investigated using Kinetic Monte Carlo simulations. Simulated nanostructures show a good agreement with those observed in Ge-Mn using Atom Probe Tomography and Transmission Electron Microscopy. Self organisation is observed although the rigid lattice simulations used do not account for misfit elastic strain. Simulations reveal that the final nanostructure, in term of number density and diameter of nanocolumns, is controlled by the early stages of growth of the film. The influence of both growth temperature and solute concentration on the nanostructure features is discussed in details. (C) 2014 AIP Publishing LLC. [http://dx.doi.org/10.1063/1.4864271]
\end{abstract}

\section{INTRODUCTION}

During the last two decades, much interest has been raised by self organized nanostructures. Nanostructures like quantum dots, nanowires, and superlattices are widely studied due to the potential applications in spintronics, optoelectronics, or data storage devices. In spintronics, the development of high Curie temperature ferromagnetic semiconductors is still a challenge for the conception of spinbased electronic devices fully compatible with the silicon technology. ${ }^{1}$ One of the most promising candidates is Germaniun-Manganese system in which elements are strongly immiscible and Curie temperature has been evidenced to be above room temperature. ${ }^{2}$ Since 2006, high Curie temperature has been observed either in diluted Mn-doped $\mathrm{Ge}^{3}$ or in nanostructured Ge-Mn films. ${ }^{2,4,5}$ Overall studies, it has been shown that Mn-rich phase can be formed in the matrix depending on the growth process and $\mathrm{Mn}$ concentration. Spherical particles ${ }^{6-9}$ with equilibrium compositions like $\mathrm{Ge}_{3} \mathrm{Mn}_{5}, \mathrm{Ge}_{8} \mathrm{Mn}_{11}$ or Mn-rich columnar phase $^{2,10-13}$ have been reported and correlated to magnetic properties. However, the origin of self organisation in columnar nanostructure observed in $\mathrm{Ge}-\mathrm{Mn}$ thin layers grown by Molecular Beam Epitaxy (MBE) is not clearly identified. The role of the misfit strain between nanocolumns and the parent phase needs to be clarified. ${ }^{14,15}$ In addition, the influence of both the growth temperature ${ }^{10,13}$ and the overall film concentration $^{10}$ on the evolution of nanostructure features (number density and size of nanocolumns) is not fully documented and understood.

The fundamental mechanism of phase separation that occurs in thin films where chemical species are immiscible is also of great concern. A two-dimensional (2D) spinodal decomposition caused by the attractive chemical pairinteraction was conjectured by Fukushima et al. in the case of low solubility of the transition-metal impurity in the semiconductors. ${ }^{16-20}$ This spinodal nano-decomposition can

a)etienne.talbot@univ-rouen.fr involve the formation of anisotropic inhomogeneities, which modify magnetic properties. ${ }^{21}$ The aim of the present work is to investigate all these key issues that are of great concern both from a fundamental point of view and also for applications in spintronics.

Much effort has been devoted to develop numerical simulations of self-organized epitaxial nanostructures. Among the different approaches, Kinetic Monte Carlo (KMC) is one of the most efficient numerical methods to simulate epitaxial growth. ${ }^{22-24}$ Simulation of phase separation in strain-free two component systems during the growth process have already been performed using KMC on a $(1+1)$-dimensional lattice mode $^{13}$ or in $3 \mathrm{D}$ with layer-by-layer ${ }^{16,25}$ or solid-on-solid growth modes. ${ }^{26}$ In the present work, the epitaxial growth of $\mathrm{A}_{\mathrm{X}} \mathrm{B}_{1-\mathrm{X}}$ layer deposited on a pure $\mathrm{A}$ substrate was investigated using rigid lattice Kinetic Monte Carlo simulations. Phase separation processes that occur in this system, where A and $\mathrm{B}$ are immiscible, were studied in details. The influence of growth temperature and chemical composition on the formation of B-rich nanocolumns (size and number density) has been studied. Simulated nanostructures were confronted to those observed in Ge-Mn thin film by Transmission Electron Microscopy (TEM) ${ }^{10,13}$ and Atom Probe Tomography $(\mathrm{APT})^{36}$ where self organisation of nanocolumns were revealed. The early stages of phase separation (nucleation of B-rich regions) are shown to control the final nanostructure.

\section{SIMULATION METHOD}

\section{A. Deposition and diffusion processes}

In order to follow the microstructure of the epitaxial growth of binary layers, Kinetic Monte Carlo simulations of deposition and diffusion processes in binary $\mathrm{A}_{\mathrm{X}} \mathrm{B}_{1-\mathrm{X}}$ alloys were performed in $2+1$ dimensions on a rigid square lattice with periodic boundary conditions in the plane directions. The simulated film was constructed by depositing atoms on a flat substrate. The substrate size is $70 a_{0} \times 70 a_{0}$ (4900 sites) where $a_{0}$ is the lattice parameter. The substrate defines $x$ and $y$ axes while $z$ axis refers to the growth direction. 
The simulation involves two fundamental events: the deposition of atoms on the substrate surface and the surface diffusion of atoms. It is important to note that the simulation was used to model the low temperature film growth, so only surface diffusion process was considered, bulk diffusion was assumed to be negligible.

Deposition event is simulated by randomly adding A or B type atoms onto the surface assuming that no vacancy exists within the film and no desorption is allowed. During deposition, all surface sites are available. The co-ordinates of the deposition site $(x, y)$ is chosen randomly and the height $(z)$ of the atomic column at the chosen $(x, y)$ coordinates is increased by one unit when the deposited atom is added. The deposition rate $W^{\text {dep }}$ is proportional to the total number of atoms reaching the substrate per unit of time. It is expressed by the number of monolayer per second and surface area. In this work, a constant incident atom flux was used and taken equal to $0.14 \mathrm{ML} / \mathrm{s}$ (estimated to fit experimental one). The deposited atoms have the possibility to diffuse onto the surface by hopping from its site to one of its nearest neighbour vacant sites. Diffusion is allowed on the four nearest neighbour sites in the plane, and lower sites at a step edge.

This thermally activated jump depends on the substrate temperature and on the local environment of the atom in both initial configuration $i$ and final configuration $f$. The jump frequency is given by

$$
W^{\text {diff }}=\nu_{0} \exp \left(-\frac{\Delta E_{i \rightarrow f}}{k_{\mathrm{B}} T}\right),
$$

where $\nu_{0}$ is the attempt frequency $\left(10^{13} \mathrm{~s}^{-1}\right), k_{\mathrm{B}}$ is Boltzmann's constant, $T$ is the growth temperature (in Kelvin), and $\Delta E_{i \rightarrow f}$ is the activation energy of the diffusion event. This activation barrier is the energy required to move the surface atom from one site to a neighbour site (Fig. 1). $\Delta E_{i \rightarrow f}$ depends on the atomic environment and may write as

$$
\Delta E_{i \rightarrow f}=E_{0}^{m}+\frac{E_{f}-E_{i}}{2},
$$

where $E_{0}^{m}(m=\mathrm{A}, \mathrm{B})$ is the energy barrier $(>0)$ for the surface migration of isolated atoms. Even if the ambition of this work is not to simulate the real system Ge-Mn, values for migration energies of $\mathrm{A}$ and $\mathrm{B}$ atoms were chosen to be realistic. As the simulated results will be compared to the microstructure observed in the Ge-Mn system, the migration energy of Ge atoms on Ge substrate was taken as reference values $^{27,28}$ for both $\mathrm{A}$ and B, i.e., $E_{0}^{A}=E_{0}^{B}=0.65 \mathrm{eV}$. $E_{i}$ and $E_{f}$ are the configuration energies $(<0)$ of the jumping atom at the initial and final states. $E_{f}-E_{i}$ is then the energy recovered by placing the atom at the final position minus the energy required to extract the jumping atom from its environment in its initial configuration. The local environment of each atom varies and so do the configuration energy and the activation barrier for surface diffusion (Fig. 1).

The cohesive model used to calculate the configuration energies is described using pair interactions on a rigid lattice. As a result, the configuration energy of the jumping atom $m$ is given by

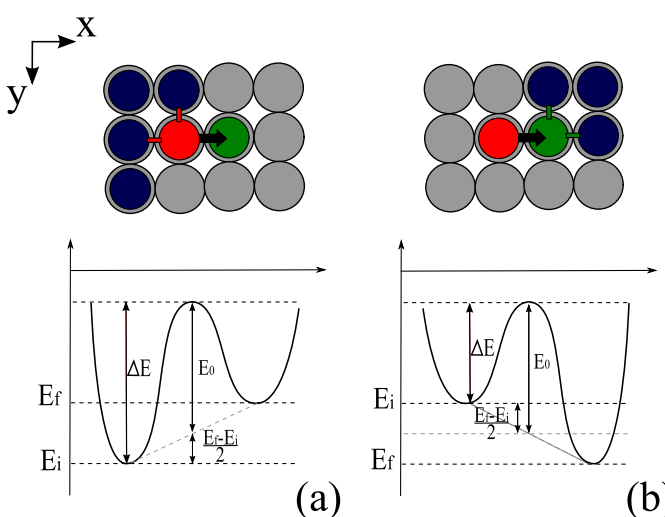

(b)

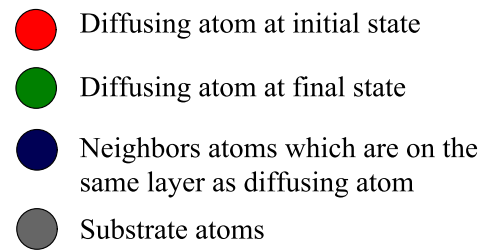

FIG. 1. Schematic energy landscape showing the dependence of the activation energy barrier with the environment of jumping species. In case (a), the initial state is more favourable than the final state and it is opposite behaviour in case (b).

$$
E=\sum_{n=A, B} n_{m n} \cdot \epsilon_{m n}
$$

where $\epsilon_{m n}(m, n=A, B)$ is the first nearest-neighbour pair energy between atoms $m$ and $n$, and $n_{m n}$ the number of bonds between the migrating atom $m$ and its neighbouring atoms (with $\sum n_{m n} \in\{1, \ldots, 5\}$ ).

In semiconductors, pair energies are around $-0.3 \mathrm{eV} .{ }^{24,29,30}$ In this work, values used are $\epsilon_{A A}=\epsilon_{B B}=$ $-0.3 \mathrm{eV}$ and $\epsilon_{A B}=-0.025 \mathrm{eV}$. Similar pair energies and ordering energy have been used by Zheng et al. ${ }^{3}$ who investigated generic epitaxial A-B layers. With these parameters, the ordering energy $\omega=\epsilon_{A B}-\frac{\epsilon_{A A}+\epsilon_{B B}}{2}$ is equal to $0.275 \mathrm{eV}$. The latter value was chosen in order to construct A-B phase diagram in which $\mathrm{A}$ is almost insoluble in $\mathrm{B}$ and vice versa for the temperature range considered in this work $(350 \mathrm{~K}-475 \mathrm{~K})$ that is much smaller than $T_{\mathrm{C}}$. Consequently, for a given composition $(X)$ of the film $\left(A_{X} B_{1-X}\right)$, the supersaturation as well as the volume fraction of nanocolumns is almost independent of temperature in this temperature range. Some authors consider an additional Ehrlich-Schwoebel barrier $^{31,32}$ to account for the case where an adatom is stepping down at a step edge. ${ }^{3,29}$ This barrier was considered as negligible in this model.

\section{B. Principle of Monte Carlo algorithm}

The simulation of the thin film growth process is based on residence time algorithm..$^{23,33,34}$ Bortz et al. ${ }^{35}$ and Maksym $^{22}$ have proposed an efficient algorithm, which ensures that each step corresponds to either deposition or diffusion process and considers groups of events instead of individual events. The algorithm computes lists of events for all deposition and diffusion phenomena. At each event $i$, a rate $W_{i}$ and a population $N_{i}$ (i.e., number of sites for which 
the event $i$ can occur) were considered. The sequence of the two following sub-steps enables to choose an individual event among all the possible ones

(1) A random number $\zeta \in] 0,1[$ is selected and the event $j$ satisfying the inequality

$$
\sum_{a=0}^{j-1} \frac{W_{a} N_{a}}{\sum_{i} W_{i} N_{i}} \leq \zeta<\sum_{a=0}^{j} \frac{W_{a} N_{a}}{\sum_{i} W_{i} N_{i}}
$$

is chosen. The event for which $a=0$ corresponds to the deposition event. Diffusion occurs for all $a>0$.

(2) A second random number $\psi$ is chosen in order to randomly select one of the sites where the event $a$ can occur (in the interval $\left[1, N_{a}\right]$ ). This sequence defines one Monte Carlo step. The physical time attached to this step is

$$
\Delta t=\left(\sum_{i} W_{i} \cdot N_{i}\right)^{-1}
$$

With this algorithm, an event (deposition or diffusion) is performed at each Monte Carlo step.

\section{RESULTS AND DISCUSSION}

\section{A. Influence of growth temperature}

Several simulations were carried out at various temperatures ranging from $350 \mathrm{~K}$ and $475 \mathrm{~K}$ and at constant incident flux of $0.14 \mathrm{ML} / \mathrm{s}$. The concentration was fixed to $\mathrm{X}_{B}=10 \%$. Let us remind that interaction parameters were chosen so as to get almost no solubility of B in A (high critical temperature $T_{\mathrm{C}}$ as in Ge-Mn system) and the precipitation of a second phase that is almost pure in B atoms. Fig. 2 presents snapshots of configurations (cross-section and plane view) for temperature ranging from $350 \mathrm{~K}$ to $450 \mathrm{~K}$. As expected, simulations exhibit the presence of nanocolumns perpendicular to the substrate. These images highlight a very important point: a well organised nanostructure is observed despite no elastic interactions between nanocolumns were considered in these simulations (Fig. 2). Plane view at $350 \mathrm{~K}$

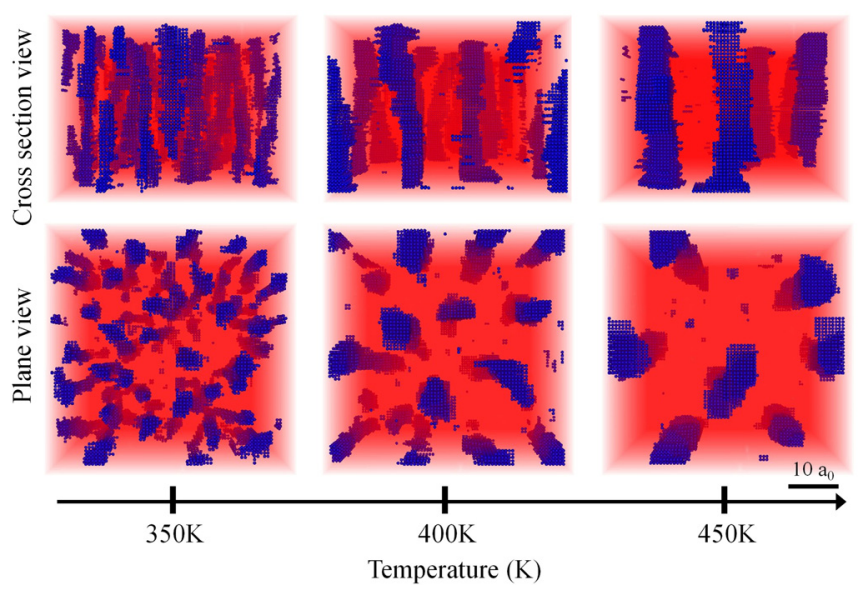

FIG. 2. Cross section view and plane view of nanostructures simulated at different growth temperatures for 10 at. \% of B atoms. (B atoms are represented by blue dots). for instance clearly shows a regular distribution of B nanocolumns, which is very similar to self organised nanostructures observed experimentally using $\mathrm{TEM}^{2,10,13}$ and Atom Probe Tomography. ${ }^{36}$ This means that the presence of elastic interactions between nanocolumns and matrix is not a necessary condition to obtain such a self-organized microstructure. A comparison between experimental and simulated nanostructure will be discussed in detail in Sec. IV.

Two main types of nanocolumns were found (Fig. 2): interrupted nanocolumns or continuous nanocolumns throughout the film. Moreover, these nanocolumns may present rippled/bent morphologies or may be completely straight. The proportion of these different types of morphologies changes with growth temperature. At low temperature $(T=350 \mathrm{~K})$, most nanocolumns are interrupted and rippled throughout the layer. At high temperatures $(T=450 \mathrm{~K})$, nanocolumns are mainly straight and mostly are uninterrupted throughout the film. Between these two temperatures (around $400 \mathrm{~K}$ ) nanocolumns are in intermediate states, i.e., majority of nanocolumns are continuous and slightly bent. It is generally in this temperature that some junctions of nanocolumns are observed.

As shown in Fig. 3, increasing the temperature from $350 \mathrm{~K}$ to $475 \mathrm{~K}$ increases the mean diameter. The surface number density of nanocolumns $\left(N_{\mathrm{S}}\right)$ is observed to decrease with $T$. The latter can be expressed as a function of a characteristic distance $\lambda$ between nanocolumns $\left(N_{\mathrm{S}}=1 / \lambda^{2}\right)$. This characteristic distance $\lambda$ is thought to be controlled by both the diffusion length and the deposition rate $(R \sim 1 / \tau$ with $\tau$ the mean time required for the deposition of a monolayer). Clearly, the mean separation between nanocolumns will increase (i.e., $N_{\mathrm{S}}$ decreases) when more time is given to the surface atoms to diffuse between each deposition events (i.e., smaller deposition rate) and when the atomic mobility is increased. Consequently, increasing temperature increases the probability for $\mathrm{B}$ atoms to reach $\mathrm{B}$ islands already formed, decreasing the formation frequency of new B-rich islands. Simulations revealed more precisely that the number density of nanocolumns decreases exponentially as a function of the reciprocal temperature (Fig. 3). Such a behaviour has been predicted theoretically and was observed during submonolayer homoepitaxial growth. ${ }^{37}$

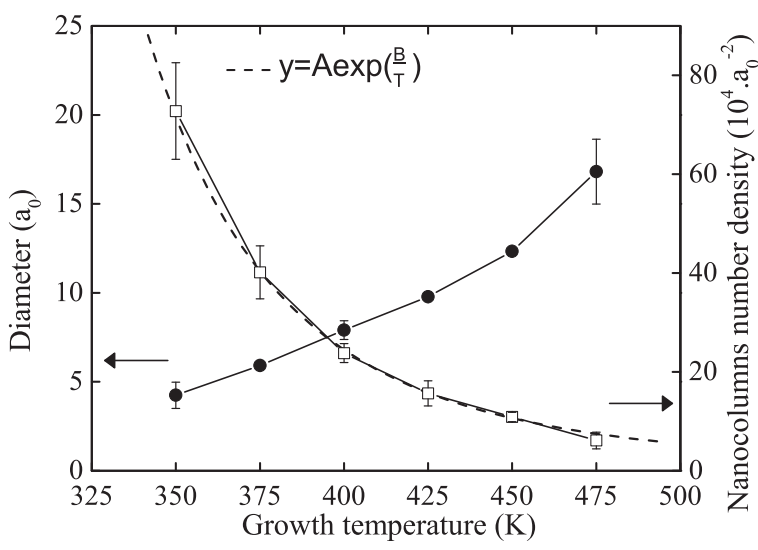

FIG. 3. Mean diameter (plain circle, expressed in unities of lattice parameter $a_{0}$ ) and number density of nanocolumns (open square) as a function of growth temperature. 
The simulations of temperature dependence performed by $\mathrm{KMC}$ are in good agreement with classical nucleation theory as well as experiments ${ }^{10,38}$ or similar simulations. ${ }^{3,25}$ However, one should keep in mind that only surface diffusion was taken into account in the present simulations. This model is therefore only applicable for fairly low temperatures, where bulk diffusion remains negligible compared to surface diffusion.

\section{B. Influence of composition}

The morphological evolution of nanocolumns was investigated for various solute atomic fractions (2 at. \% to 12 at. \% of B atoms). Section III A has shown that the growth temperature is a crucial parameter as it controls the ratio between diffusion jumps and deposition events. Therefore, two growth temperatures were considered ( $400 \mathrm{~K}$ and $450 \mathrm{~K}$ ).

Simulated nanostructures at $400 \mathrm{~K}$ and $450 \mathrm{~K}$ for different solute concentrations are provided in Fig. 4. Clearly and as already discussed in Sec. III A, the number density of nanocolumns appears much smaller for the higher temperature $(450 \mathrm{~K})$ and their diameter is also larger. At low concentration of solute $(2$ at. \% B) and at both temperatures, nanocolumns are fragmented. When the concentration increases, nanocolumns become larger and the length of fragments increases up to reach a maximum size of the film thickness. Nevertheless, different morphologies can be observed as a function of the growth temperature (see Sec. III A). At high temperature $(T=450 \mathrm{~K})$, increasing the concentration leads to well separated and completely straight nanocolumns. In contrast at low temperature $(T=400 \mathrm{~K})$, increasing solute concentration leads to rippled/interconnection nanocolumns for concentration between 2 at. $\%$ and 10 at. $\%$ of B atoms. At high B concentration, nanocolumns are well separated. Similar nanostructure evolution with the concentration has been observed using TEM. ${ }^{10,13}$

The mean diameter and the density of nanocolumns derived from simulations are presented in Fig. 5. and compared to experimental data. ${ }^{10}$ One observes, in Fig. 5(a)), an increase in mean diameter with B content. This evolution can be easily accounted for by the increase in driving force

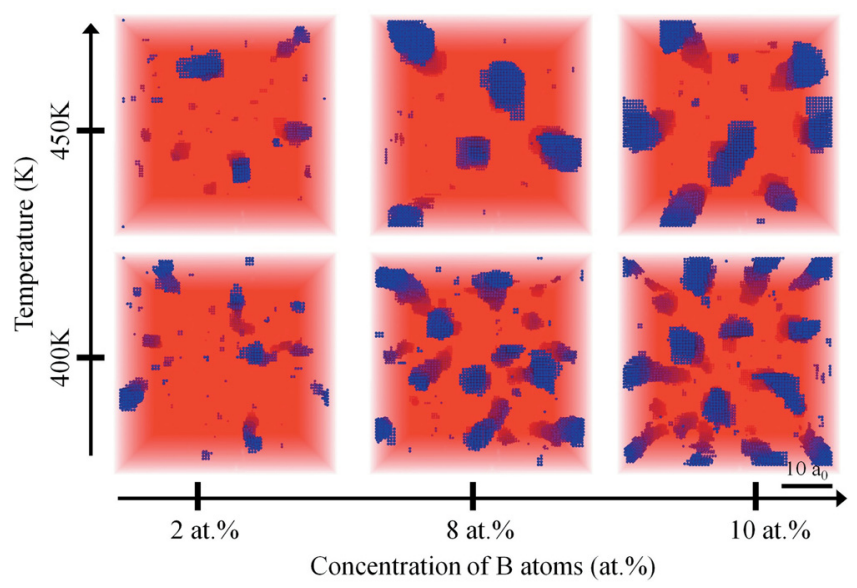

FIG. 4. Plane view of nanostructures simulated for various solute concentrations and at growth temperature of $400 \mathrm{~K}$ and $450 \mathrm{~K}$. (B phase is represented by blue dot).
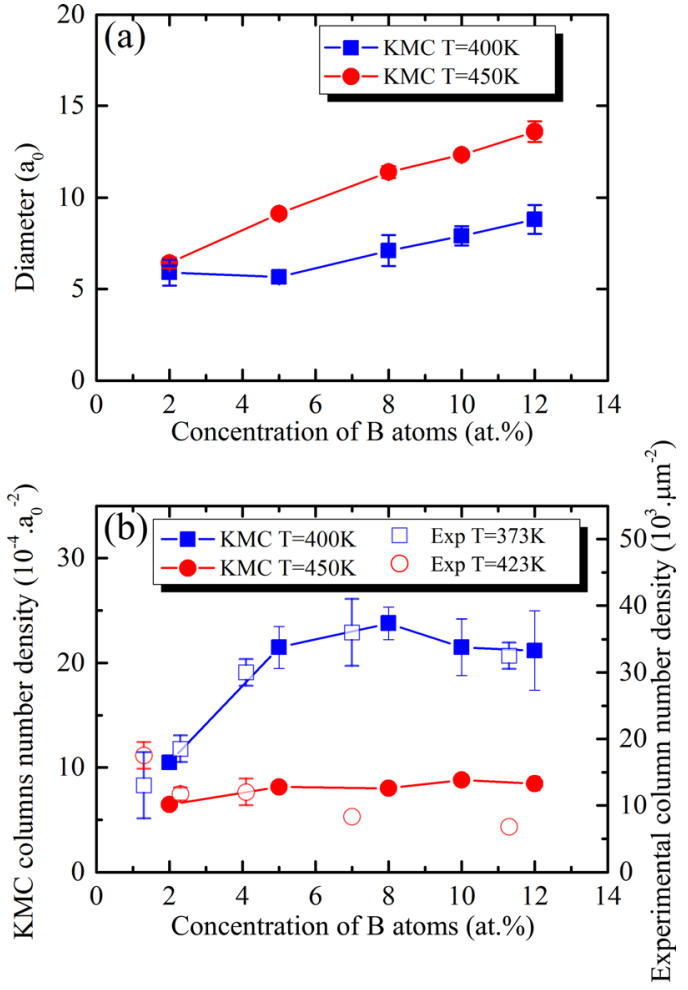

FIG. 5. (a) mean diameter of nanocolumns (in unities of lattice parameter $a_{0}$ ) as a function of B concentration at $T=400 \mathrm{~K}$ (blue square) and $450 \mathrm{~K}$ (red circle)). (b) Evolution of the number density of nanocolumns with solute concentration at $400 \mathrm{~K}$ (blue plain square) and $450 \mathrm{~K}$ (red plain circle) as derived from KMC simulations and TEM experiments of Devillers et al. ${ }^{10}$ (open symbol).

for growth with the increase in B concentration. The influence of $\mathrm{B}$ concentration on the evolution of the mean diameter is more pronounced at higher temperature because of the higher diffusivity. Regarding the evolution of the number density of nanocolumns in Fig. 5(b), it must be emphasised that simulation well reproduces the experimental evolutions obtained by Devillers et al. by $\mathrm{TEM}^{10}$ at both low and high temperatures. In both simulation and experiment cases, two distinct behaviours are observed depending on the temperature. At higher temperature $(450 \mathrm{~K})$, simulations revealed that the number density only slowly increases with B content in agreement with TEM experiments. The size of nanocolumns increases almost linearly with concentration. At lower temperature $(400 \mathrm{~K})$, the diameter of nanocolumns only increases for concentrations exceeding 5 at. \%. One can note that counting the number density for such low B content is a hard task because of their small dimensions (lateral and longitudinal). This could account for a part of the discrepancies observed between simulations and experiments. At low temperature, for both experiment and simulation, the number density evolves significantly with B content up to about 5 at. $\%$ of B and reaches a plateau for higher B content. To the authors' knowledge, this behaviour remains an open question.

To understand this latter point, the early stages of growth of the binary film were studied. Especially, the evolution of the island density with coverage rate and the influence of composition were investigated. In these simulations, 
a substrate size of $222 a_{0} \times 222 a_{0}$ (around ten times larger than previously) was used to reach better statistics. Nucleation and growth of islands is well understood and widely described in literature in case of homoepitaxy growth. ${ }^{37,39}$ However, there are only few studies dealing with binary alloys. ${ }^{40,41}$ Fig. $6(\mathrm{~b})$ presents a typical variation of the number density of islands (respectively A rich and B rich) as a function of the coverage at $T=400 \mathrm{~K}$ for 10 at. \% of $\mathrm{B}$. The behaviour of A islands density is very close to the evolution observed in homoepitaxy. ${ }^{39}$ This is probably because the film is mainly composed of A atoms $\left(X_{A}=90 \%\right)$. Regarding the island densities, obviously the density of B island is much lower than A's but more importantly its evolution with coverage follows a different trend. The film growth can be divided into four distinct regimes (Fig. 6(b)).

- The first regime corresponds to low coverage $(\theta<0.2$ ML): $A$ and B islands are isolated on the surface (Fig. 6(a) for $\theta=0.1 \mathrm{ML}$ ) and their density increases with coverage. At the very beginning of the film growth, A or B adatoms population on the surface is important, the probability that an adatom meets another one is high. This first leads to the formation of a dimer, a trimer, and a new island. With

(a)
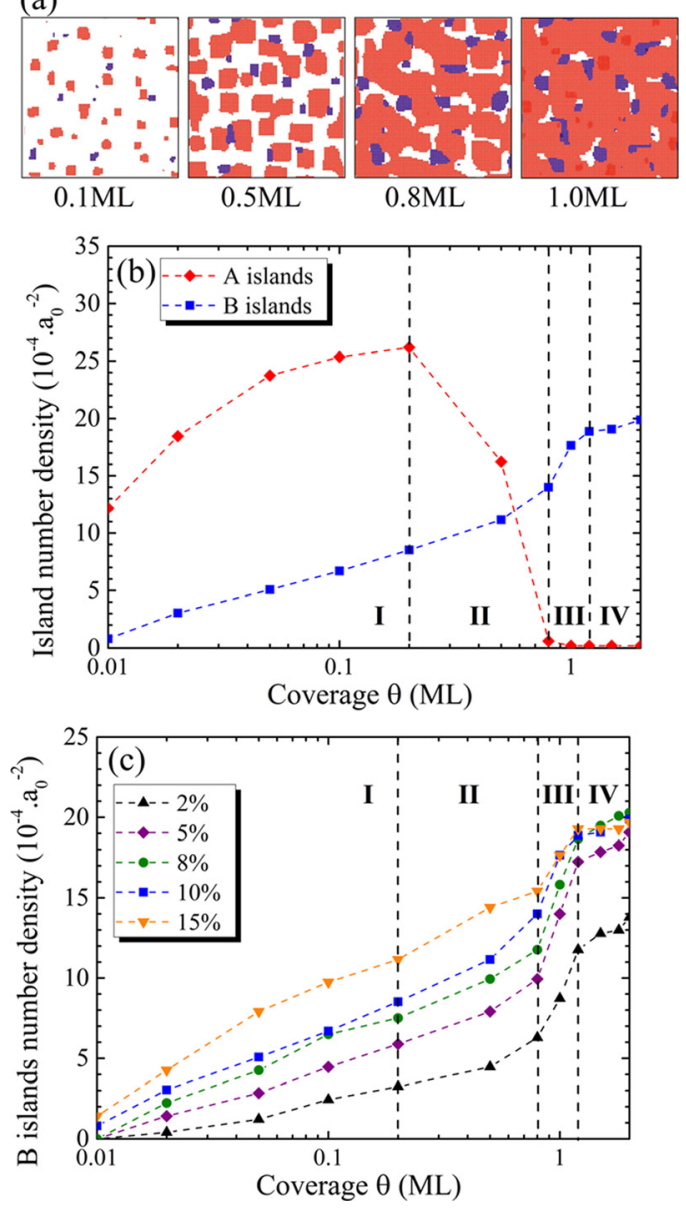

FIG. 6. (a) Snapshots of the surface morphology as function of the coverage for binary alloy $\mathrm{A}_{0.90} \mathrm{~B}_{0.10}$ at $T=400 \mathrm{~K}$. (b) Number density of $\mathrm{A}$ and $\mathrm{B}$ islands as a function of the coverage for binary alloy $\mathrm{A}_{0.90} \mathrm{~B}_{0.10}$ at $T=400 \mathrm{~K}$. (c) Number density of B islands as a function of the coverage for increasing B content from 2 at. $\%$ to 15 at. $\%(T=400 \mathrm{~K})$. increasing islands density, the probability that an A adatom is captured by an existing island becomes higher and higher at the expense of the new island formation probability.

- In the second regime $(0.2 \mathrm{ML}<\theta<0.8 \mathrm{ML})$, the evolutions of the number densities of $\mathrm{A}$ and $\mathrm{B}$ islands are very different. In proportion as the coverage is increased, the A island density significantly decreases. This is the result of A islands coalescence (Fig. 6(a) for $\theta=0.5 \mathrm{ML}$ ). To the contrary, B island density continues to slowly increase due to nucleation of new B islands. At the end of this regime, coalescence of $\mathrm{A}$ islands leads to percolation and the formation of a single interconnected zone composed of $\mathrm{A}$ atoms. In literature, the site percolation threshold of square lattices is estimated around $0.6,{ }^{28,29}$ which is in good agreement with our simulation (between 0.5 and 0.8 ).

- In the third regime $(0.8 \mathrm{ML}<\theta<1.2 \mathrm{ML})$, there is an interconnected network of A but the first layer is still not complete (Fig. 6(a) for $\theta=0.8 \mathrm{ML}$ ). The open area in the interconnected structure (A atoms) forms short channels and holes. For a coverage between $0.8 \mathrm{ML}$ and $1.2 \mathrm{ML}$ (Fig. 6(a) for $\theta=1.0 \mathrm{ML}$ ), the most probable site for one atom to be deposited is on the top of the A interconnected zone (i.e., on the second layer). Then adatoms diffuse on the surface until they either nucleate/extend an island on the second layer or fall down in an open space. As most of the step edges are composed of A atoms, when A atoms fall in an open space, they diffuse along the step and extend the A atoms interconnected network. It is more complicated for B adatoms. B atoms are almost insoluble in A. Consequently, for a B fallen atom, the short channels or holes constitute confined spaces. In case of no possibility to form dimer or to join an existing island (i.e., if no B islands is located around the hole or short channel), the B fallen atom will constitute a new site for nucleation of island. This leads to a significant increase in number density of $\mathrm{B}$ islands. This regime stops at $1.2 \mathrm{ML}$, when the first layer is complete and then the number density of B islands becomes almost constant.

- At the beginning of the last stage, from 1.2 ML, the first monolayer is complete and some islands of A and B atoms already exist on the second monolayer leading to a slight surface roughness. This complete layer will not change anymore in the framework of the present model. At this stage, it is worth noting that major differences exist between the first and the second layer growth: in the first layer, atoms are deposited on a pure A substrate whereas in the second layer, B islands already exist. Consequently, in the latter case, energetically favourable sites for deposited B atoms are already present. So, B deposited atoms diffuse up to reach pre-exisiting $B$ islands and contribute to the vertical growth of islands formed in the first layer and thus to the vertical growth of nanocolumns. The number density of $\mathrm{B}$ islands thus controls the number density of nanocolumns finally observed. This is therefore the early stages of formation of B-islands that control the subsequent layer by layer evolution, and the final nanostructure of the thin film. 
The challenge is now to understand why the number density of $\mathrm{B}$ island reaches a plateau when increasing the concentration above 5 at. \% B (Fig. 5). Fig. 6(c) presents the variation of $\mathrm{B}$ atoms island number density with coverage for different film compositions (from 2 to 15 at. \% B). This figure shows that

- Whatever the composition, the fourth regimes are observed for the same coverage values.

- During stage I and II, the slope is observed to increase with concentration $(\mathrm{X})$. When the concentration of solute increases, it is less and less difficult and time consuming for the diffusing B adatoms to meet another B atom and to form a dimer, a trimer, and in-fine a super-critical nucleus.

- During stage III, the number density of B islands increases drastically with coverage. For higher concentrations, this increase is less pronounced. As explained previously, this is due to confinement of $\mathrm{B}$ atoms into open area of the interconnected structure of $\mathrm{A}$ islands. At the beginning of this stage, higher the concentration, higher the number density of $\mathrm{B}$ islands. The probability that a confined $\mathrm{B}$ adatom joins already present $B$ islands increases at the expense of the nucleation of a new island.

- In stage IV, the islands density of B atoms for concentration ranging from 5 to 15 at. \% reaches almost the same value whereas for 2 at. \% this value is lower. This observation is in a good agreement with the plateau observed in Fig. 5(b).

Let us come back to the plateau observed in Fig. 5(b). For higher concentrations, the number density of islands is so high that adatoms can easily join already formed islands so that no new B islands nucleate. Increasing temperature will evidently exaggerate this trend. In homoepitaxy, it is known that this maximum number density is dependant on the ratio between deposition flux and surface diffusion coefficients. ${ }^{42,43}$ It is anticipated that we will have similar behaviour here although more complex with a dependency with concentration. Modelling this behaviour is beyond the scope of the present paper.

\section{Nanocolumn morphology}

Simulations have been compared to APT experiments dealing with on $\mathrm{Ge}_{0.9} \mathrm{Mn}_{0.1}$ thin films grown at $100^{\circ} \mathrm{C}$ (Ref. 36) (Fig. 7). Mn-enriched nanocolumns embedded in a pure Ge-matrix were evidenced. Note that the measured Mn concentration in nanocolumns was about $30 \%,{ }^{36}$ in contrast to simulated nanocolumns that are pure in B atoms. Again, nanocolumns appear well organised both in simulations and APT experiments. The experimental number density of nanocolumns was found higher than in simulation. This is expected because nanocolumns contain $\sim 30$ at. \% of $\mathrm{Mn}$ instead of 100 at. \% of B for simulation. As a result, the surface fraction (i.e., molar fraction $f \sim X / X_{\beta}$ ) of nanocolumns is higher in experiments, leading therefore to a larger number density $\left(f=\pi r^{2} N_{\mathrm{S}}\right)$.

Simulations reproduce strikingly well morphologies observed experimentally although no elastic strains were considered in the model. Nevertheless simulated

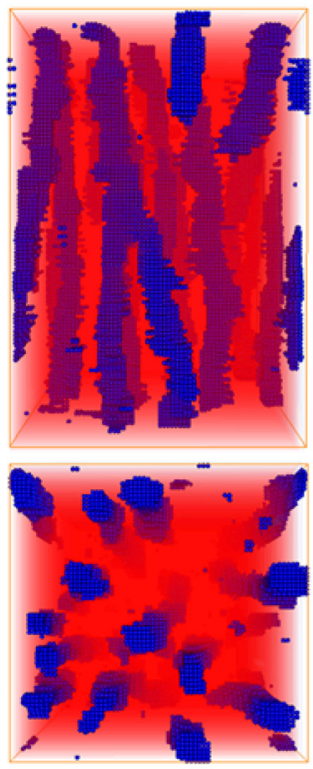

(a) $(70 \times 70 \times 150$ sites $)$

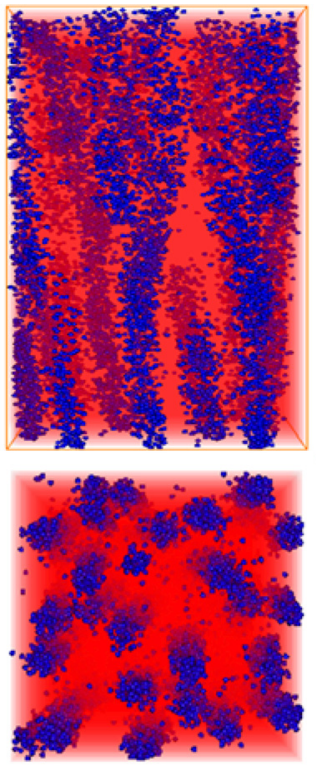

(b) $\left(20 \times 20 \times 42 \mathrm{~nm}^{3}\right)$
FIG. 7. Comparison of atomic maps as obtained by Kinetic Monte Carlo simulations (a) and atom probe tomography experiments (b). (a) Generic binary alloys $\mathrm{A}_{0.90} \mathrm{~B}_{0.10}$ were simulated using Kinetic Monte Carlo at $\mathrm{T}=400 \mathrm{~K}$ (b). 3D reconstruction of the atomic distribution of $\mathrm{Ge}$ and $\mathrm{Mn}$ atoms in $\mathrm{A}_{0.90} \mathrm{~B}_{0.10}$ thin layers as obtained by atom probe tomography.

nanocolumns appear less well defined compared to experiments. Elastic strains originating from the lattice misfit between nanocolumns and the parent were not taken into account in simulations. However, their influence on the nanostructuration appears small as self organisation is reproduced in simulation. Thermodynamics and surface diffusion considerations appear sufficient to reproduce experimental nanostructures (Fig. 7). Characteristic length appears to be controlled by the mobility ( $D$ the diffusion coefficient) and the deposition rate $\left(\lambda \sim(D \tau)^{1 / 2}\right.$ with $\tau$ being the characteristic time for the deposition of a monolayer (related to deposition rate)).

During layer by layer growth, some interrupted or new nanocolumns may form. These two types of nanocolumns can be explained by the presence of A interconnected zone /open spaces (defined in Sec. IIIB) on each layer during growth.

- New nucleation sites may appear in open spaces existing in the second layer or subsequent layers, leading to new nanocolumns.

- Nanocolumns may be bent/interrupted by the extension of the A interconnected zone partially/completely over the existing B islands or nanocolumns.

- During growth, interconnected or split up nanocolumns (Y-shape) may form respectively when two bent nanocolumns meet or when A interconnected zones spread over B islands.

\section{CONCLUSION}

We have investigated the formation of nanocolumns during the epitaxial growth of immiscible $\mathrm{AB}$ surface alloy 
using Kinetic Monte Carlo method and we have compared simulations to experimental results on Ge-Mn systems. Results indicate that self organization is reproduced in simulation without any elastic interaction. Our results are able to explain the evolution of B-rich columns density, which converges toward a maximum density regardless of $B$ concentration. This phenomenon has been interpreted in terms of growth of $\mathrm{A}$ islands and confinement of $\mathrm{B}$ species during the growth of the first monolayer. The simple simulation approach presented in this paper leads to results in good agreement with experimental TEM and APT results on Ge-Mn systems. This work suggests that thermodynamics and surface diffusion are the most predominant mechanism, which control the self-organization of magnetic Ge-Mn nanocolumns.

\section{ACKNOWLEDGMENTS}

Simulations were performed at the Centre de Ressources Informatiques de Haute-Normandie (CRIHAN) under Project No. 2012003.

${ }^{1}$ I. Zutic, J. Fabian, and S. Das Sarma, Rev. Mod. Phys. 76, 323 (2004).

${ }^{2}$ M. Jamet, A. Barski, T. Devillers, V. Poydenot, R. Dujardin, P. BayleGuillemaud, J. Rothman, E. Bellet-Amalric, A. Marty, J. Cibert, R. Mattana, and S. Tatarenko, Nature Mater. 5, 653 (2006).

${ }^{3}$ S. Zheng, W. Zhu, Y. F. Gao, G. M. Stocks, and Z. Zhang, Appl. Phys. Lett. 96, 071913 (2010).

${ }^{4}$ Y. J. Cho, C. H. Kim, H. S. Kim, W. S. Lee, S.-H. Park, J. Park, S. Y. Bae, B. Kim, H. Lee, and J.-Y. Kim, Chem. Mater. 20, 4694 (2008).

${ }^{5}$ F. Xiu, Y. Wang, J. Kim, A. Hong, J. Tang, A. P. Jacob, J. Zou, and K. L. Wang, Nature Mater. 9, 337 (2010).

${ }^{6}$ C. Bihler, C. Jaeger, T. Vallaitis, M. Gjukic, M. S. Brandt, E. Pippel, J. Woltersdorf, and U. Gosele, Appl. Phys. Lett. 88, 112506 (2006).

${ }^{7}$ Y. Wang, J. Zou, Z. Zhao, X. Han, X. Zhou, and K. L. Wang, Appl. Phys. Lett. 92, 101913 (2008).

${ }^{8}$ S. Ahlers, D. Bougeard, N. Sircar, G. Abstreiter, A. Trampert, M. Opel, and R. Gross, Phys. Rev. B 74, 214411 (2006).

${ }^{9}$ J.-P. Ayoub, L. Favre, I. Berbezier, A. Ronda, L. Morresi, and N. Pinto, Appl. Phys. Lett. 91, 141920 (2007).

${ }^{10}$ T. Devillers, M. Jamet, A. Barski, V. Poydenot, P. Bayle-Guillemaud, E. Bellet-Amalric, S. Cherifi, and J. Cibert, Phys. Rev. B 76, 205306 (2007).

${ }^{11}$ D. Bougeard, S. Ahlers, A. Trampert, N. Sircar, and G. Abstreiter, Phys. Rev. Lett. 97, 237202 (2006).

${ }^{12}$ F. Xiu, Y. Wang, K. Wong, Y. Zhou, X. Kou, J. Zou, and K. L. Wang, Nanotechnology 21, 255602 (2010).

${ }^{13}$ Y. Wang, F. Xiu, Y. Wang, X. Kou, A. P. Jacob, K. L. Wang, and J. Zou, J. Alloys Compd. 508, 273 (2010).
${ }^{14}$ S. Tardif, V. Favre-Nicolin, F. Lanon, E. Arras, M. Jamet, A. Barski, C. Porret, P. Bayle-Guillemaud, P. Pochet, T. Devillers, and M. Rovezzi, Phys. Rev. B 82, 104101 (2010).

${ }^{15}$ E. Prestat, A. Barski, E. Bellet-Amalric, J.-F. Jacquot, R. Morel, D. Tainoff, A. Jain, C. Porret, P. Bayle-Guillemaud, and M. Jamet, Appl. Phys. Lett. 103, 012403 (2013).

${ }^{16}$ T. Fukushima, K. Sato, H. Katayama-Yoshida, and P. H. Dederichs, Jpn. J. Appl. Phys., Part 2 45, L416 (2006).

${ }^{17}$ K. Sato, H. Katayama-Yoshida, and P. H. Dederichs, Jpn. J. Appl. Phys., Part 2 44, L948 (2005).

${ }^{18}$ H. Katayama-Yoshida, K. Sato, T. Fukushima, M. Toyoda, H. Kizaki, V. A. Dinh, and P. H. Dederichs, Phys. Status Solidi A 204, 15 (2007).

${ }^{19}$ H. Katayama-Yoshida, K. Sato, T. Fukushima, M. Toyoda, H. Kizaki, V. A. Dinh, and P. H. Dederichs, J. Magn. Magn. Mater. 310, 2070 (2007).

${ }^{20}$ K. Sato, L. Bergqvist, J. Kudrnovsky, P. H. Dederichs, O. Eriksson, I. Turek, B. Sanyal, G. Bouzerar, H. Katayama-Yoshida, V. A. Dinh, T. Fukushima, H. Kizaki, and R. Zeller, Rev. Mod. Phys. 82, 1633 (2010).

${ }^{21}$ K. Sato, T. Fukushima, and H. Katayama-Yoshida, Jpn. J. Appl. Phys., Part 2 46, L682 (2007).

${ }^{22}$ P. A. Maksym, Semicond. Sci. Technol. 3, 594 (1988).

${ }^{23}$ A. C. Levi and M. Kotrla, J. Phys. Condens. Matter 9, 299 (1997)

${ }^{24}$ S. Clarke, M. R. Wilby, and D. D. Vvedensky, Surf. Sci. 255, 91 (1991).

${ }^{25}$ J. H. He, C. A. Carosella, G. K. Hubler, S. B. Qadri, and J. A. Sprague, Phys. Rev. B 73, 235406 (2006).

${ }^{26}$ J. Otomo, R. Kurokawa, H. Takahashi, and H. Nagamoto, Vacuum 81, 1003 (2007).

${ }^{27}$ K. A. Bratland, Y. L. Foo, J. A. N. T. Soares, T. Spila, P. Desjardins, and J. E. Greene, Phys. Rev. B 67, 125322 (2003).

${ }^{28}$ S. J. Chey, J. E. Van Nostrand, and D. G. Cahill, Phys. Rev. Lett. 76, 3995 (1996).

${ }^{29}$ F. Elsholz, M. Meixner, and E. Schll, Nucl. Instrum. Methods B 202, 249 (2003).

${ }^{30}$ M. Mixa, V. Hol, G. Springholz, and G. Bauer, Phys. Rev. B 80, 045325 (2009).

${ }^{31}$ G. Ehrlich and F. G. Hudda, J. Chem. Phys. 44, 1039 (1966).

${ }^{32}$ R. L. Schwoebel and E. J. Shipsey, J. Appl. Phys. 37, 3682 (1966).

${ }^{33}$ S. Lucas and P. Moskovkin, Thin Solid Films 518, 5355 (2010).

${ }^{34}$ W. M. Young and E. W. Elcock, Proc. Phys. Soc. 89, 735 (1966).

${ }^{35}$ A. B. Bortz, M. H. Kalos, and J. L. Lebowitz, J. Comput. Phys. 17, 10 (1975).

${ }^{36}$ I. Mouton, R. Lard, E. Talbot, E. Cadel, C. Genevois, D. Blavette, V. Baltz, E. Prestat, P. Bayle-Guillemaud, A. Barski, and M. Jamet, J. Appl. Phys. 112, 113918 (2012).

${ }^{37}$ J. A. Venables, G. D. T. Spiller, and M. Hanbucken, Rep. Prog. Phys. 47, 399 (1984).

${ }^{38}$ A. Jain, M. Jamet, A. Barski, T. Devillers, C. Porret, P. Bayle-Guillemaud, S. Gambarelli, V. Maurel, and G. Desfonds, Appl. Phys. Lett. 97, 202502 (2010).

${ }^{39}$ J. G. Amar, F. Family, and P.-M. Lam, Phys. Rev. B 50, 8781 (1994).

${ }^{40}$ M. Einax, S. Ziehm, W. Dieterich, and P. Maass, Phys. Rev. Lett. 99, 016106 (2007).

${ }^{41}$ W. Dieterich, M. Einax, and P. Maass, Eur. Phys. J. Spec. Top. 161, 151 (2008).

${ }^{42}$ T. Irisawa, Y. Arima, and T. Kuroda, J. Cryst. Growth 99, 491 (1990).

${ }^{43}$ J. Villain, A. Pimpinelli, L. Tang, and D. Wolf, J. Phys. I France 2, 2107 (1992). 